\title{
Desempenho operacional e custos de um trator na irrigação pós-plantio de eucalipto em campo
}

\author{
Danilo Simões ${ }^{1}$, Magali Ribeiro da Silva ${ }^{2}$
}

\begin{abstract}
RESUMO
O alto investimento em operações agroflorestais mecanizadas implica a definição segura de qual será a máquina mais preconizada para a otimização da operação, vinculada aos menores custos envolvidos. Este estudo teve como objetivo avaliar o desempenho operacional e econômico da operação silvicultural de irrigação pós-plantio de eucalipto a campo. A análise técnica englobou um estudo de tempos e movimentos e, a análise econômica, as variáveis do custo operacional, custo de produção e rendimento energético. Os resultados obtidos evidenciaram que, para áreas de reflorestamento de eucalipto em primeira e terceira rotação, não há uma diferença da capacidade de campo efetiva para a operação de irrigação pós-plantio. Em decorrência do dispêndio com tempos improdutivos, houve uma redução significativa na capacidade de campo operacional e, consequentemente, uma elevação no custo de produção.
\end{abstract}

Palavras-chave: máquinas agrícolas, silvicultura, sobrevivência de mudas.

\begin{abstract}
Operating performance and costs of a tractor in irrigation after eucalyptus planting in the field

The high investment in mechanized agroforestry operations involves the secure definition of which machine will be the most recommended for optimization of the operation associated to the lowest costs involved. The aim of this research was to study the operational and economical performance of the silvicultural operation of irrigation after planting of eucalyptus in the field. The technical analysis included a study on time and movements and the economic analysis, variables of operational cost, production cost and energy consumption. The results showed that for areas of eucalypt plantations in the first and third rotation, there is no difference in the effective field capacity for the operation of irrigation after planting. Due to result of expenditure on unproductive times, there was a significant reduction in the operational field capacity and hence an increase in production cost.
\end{abstract}

Key words: agricultural machines, silviculture, seedling survival.

Recebido para publicação em 12/05/2011 e aprovado em 09/04/2012

${ }^{1}$ Administrador de Empresas. Mestre, Doutorando do Programa de Pós-graduação em Agronomia - Energia na Agricultura, Depto. de Recursos Naturais/Ciências Florestais, Universidade Estadual Paulista (UNESP), Caixa Postal 237, 18610-307 Botucatu, São Paulo, Brasil. simoesdanilo@yahoo.com.br *Autor correspondente

${ }^{2}$ Engenheira Florestal, Doutora. Departamento de Recursos Naturais/Ciências Florestais, Universidade Estadual Paulista (UNESP), Caixa Postal 237, 18610-307, Botucatu, São Paulo,Brasil.magaliribeiro@fca.unesp.br 


\section{INTRODUÇÃO}

O preparo da área e do solo para o plantio de espécies florestais objetiva disponibilizar quantidade suficiente de água e nutrientes para o mais rápido estabelecimento das mudas. Em geral, as técnicas de preparo, além de visarem ao rápido crescimento do sistema radicular, por meio do revolvimento mais ou menos localizado do solo, facilitam a absorção de água e de nutrientes e, também eliminam plantas indesejáveis próximas das mudas da espécie florestal, evitando a competição (Gatto et al., 2003).

A irrigação de mudas de essências florestais, durante o plantio e nas primeiras semanas da implantação, é uma operação importante nos plantios comerciais, principalmente nas épocas secas do ano, influindo na sobrevivência e desenvolvimento das mudas (Buzetto, 2002). Além disso, a capacidade de armazenamento de água de alguns solos de textura mais arenosa pode ser um fator limitante ao plantio (Kramer, 1983, citado por Buzetto, 2002). Stape (1989), citado por Gruber (2006), concluiu que o volume de $6 \mathrm{~L}$ por planta e por irrigação (aplicados um dia antes do plantio, logo após o plantio e depois de uma semana) foi o suficiente para reduzir os custos com replantio e tornar o desenvolvimento das mudas mais homogêneo.

Segundo Simões (1989), a qualidade das mudas refletese no crescimento futuro das árvores e, portanto, pode interferir na produtividade da floresta. Para se ter uma floresta de boa qualidade e que atenda às necessidades da humanidade é preciso mudas sadias e bem desenvolvidas. Para isso, cada vez mais se faz necessário o uso da irrigação. Constituindo uma técnica que proporciona alcançar a máxima produção, em complementação às demais práticas agrícolas, a irrigação tem sido alvo de considerável interesse por parte dos silvicultores (Lima et al., 2004).

O fornecimento de água para as mudas de eucalipto após o plantio é característico da prática silvicultural, para assegurar um bom índice de sobrevivência das mudas. Entretanto, é uma prática que tem elevado custo operacional, dentre as demais atividades de implantação de florestas.

De acordo com Silva et al. (2004), as informações relativas aos custos de todas as etapas de implantação de um projeto são extremamente necessárias para a viabilização de recursos para a execução de cada fase. Peloia \& Milan (2010) afirmam que a mecanização agrícola no Brasil representa um fator de grande importância para a competitividade em termos de custo, chegando a ser o segundo fator de produção mais importante, sendo inferior apenas à posse da terra. Em termos de potencial para redução dos custos de produção, a mecanização pode ser considerada como o fator principal. Molin et al., (2006) complementam que as informações sobre o desempenho e a capacidade de trabalho das máquinas agrícolas são de grande impor- tância no gerenciamento de sistemas mecanizados agrícolas, auxiliando na tomada de decisões. A obtenção de informações sobre o desempenho é normalmente realizada de modo manual, por meio da análise dos tempos e movimentos.

Nesse sentido, o experimento foi realizado com o objetivo de avaliar o desempenho operacional e econômico do trator agrícola, na operação de irrigação pós-plantio de eucalipto a campo, em distintas áreas de reflorestamento.

\section{MATERIAL E MÉTODOS}

A área experimental está localizada no sul do Estado da Bahia. Segundo Embrapa (1999), o solo da área é um "Latossolo Amarelo álico, coeso, relevo plano", classificado como Latossolo Amarelo coeso, LAx. A área estudada, segundo a classificação de Wilhelm Köeppen, está localizada em área de clima Af (Bahia, 1998), tropical chuvoso de floresta, com 1 a 3 meses secos. O total de precipitação pluvial anual apresenta a média de $1.587,5 \mathrm{~mm}$. A temperatura média anual é de $24,5^{\circ} \mathrm{C}$.

Com o propósito de atingir a finalidade do estudo, foi necessário fixar os diversos fatores que interferem no desempenho operacional da irrigação pós-plantio a campo, ou seja, estudou-se a irrigação sob condições homogêneas de solo, declividade, clima, local, máquina e operador. Entretanto, para avaliar se há influência do número de reformas do povoamento florestal, a área experimental foi subdividida em 2 tratamentos, sendo esses:

Tratamento 1: área de reflorestamento de eucalipto, com 16,71 ha, após manejado por talhadia, por três rotações, com linhas de plantio estabelecidas por meio do implemento agrícola ripper, acoplado a um trator com rodados de esteiras;

Tratamento 2: área de reflorestamento de eucalipto, com 15,16 ha, após manejado por talhadia, por uma rotação, com linhas de plantio estabelecidas por meio do implemento agrícola subsolador, acoplado a um trator com rodados de pneus.

O trator agrícola empregado no estudo era da marca John Deere - modelo 5605, equipado com motor diesel marca John Deere - modelo 4045D, com potência do motor na rotação nominal de $55 \mathrm{~kW}(75 \mathrm{cv})$ e peso total de $4.000 \mathrm{~kg}$. Os pneus dianteiros eram 12.4-24 R1, com 8 lonas e, os traseiros, 16.9-30 R1, com 12 lonas, lastrados com pesos metálicos. A atividade foi operada em terceira marcha reduzida, com a tração dianteira auxiliar (TDA) acionada, com a rotação do motor fixada em 1500 rotações por minuto, num eito de irrigação composto por três linhas de plantio, com espaçamento de $3 \times 3$ metros.

O implemento agrícola utilizado para a irrigação foi um tanque pipa, com capacidade de armazenamento para 5.000 
litros de água, com três mangueiras de 0,0508 m de diâmetro e válvulas nas extremidades para controle da dosagem da água, conduzidas por três ajudantes florestais, permitindo a aplicação de três litros de água por muda de eucalipto. A irrigação pós-plantio a campo foi desempenhada no mesmo dia em que se realizou o plantio das mudas de eucalipto.

A coleta dos dados de tempos e movimentos foi efetuada pelo método de cronometragem de tempo contínuo. Esse método caracteriza-se pela medição do tempo sem detenção do cronômetro, isto é, de forma contínua (Simões \& Silva, 2010). O número de ciclos operacionais foi estimado de acordo com o método proposto por Barnes (1968). Por meio de um estudo-piloto, foi obtido o número mínimo de ciclos operacionais, para um erro de amostragem admissível fixado em 5, a 95\% de probabilidade (Equação 1).

$\mathrm{n} \geq \frac{\mathrm{t}^{2} C V^{2}}{E^{2}}$

em que:

n - número mínimo de ciclos operacionais necessários;

$\mathrm{t}$ - valor de t, Student, na probabilidade desejada e (n-1) graus de liberdade;

$\mathrm{CV}$ - coeficiente de variação (\%);

E-erro admissível (\%).

A disponibilidade mecânica, segundo Simões \& Fenner (2010), é definida como o percentual do tempo de trabalho, delineado à máquina mecanicamente apta a desenvolver suas operações, o qual consiste em desconsiderar o tempo despendido para efetuar reparos ou manutenção (Equação 2).

$D m=\frac{T_{\text {int }}}{T_{\text {tot }}} 100$

em que:

Dm-grau de disponibilidade mecânica (\%);

$\mathrm{T}_{\text {int }}$-tempo de interrupção para efetuar reparos ou manutenção (h);

$\mathrm{T}_{\text {tot }}$-tempo total de trabalho (horas).

A eficiência de utilização apresenta equivalência com relação às horas utilizadas e as horas totais, por conseguinte, advém do tempo improdutivo da máquina agrícola (Equação 3).

$E u=\frac{T_{p r o}+T_{a u x}}{T_{p r o}+T_{a u x}+T_{i m p}+T_{\mathrm{int}}}$

em que:

$\mathrm{Eu}-$ eficiência de utilização (\%);
$\mathrm{T}_{\text {pro }}$ - tempo produtivo (h);

$\mathrm{T}_{\text {aux }}$ - tempo auxiliar (h);

$\mathrm{T}_{\text {imp }}$-tempo improdutivo (h);

$\mathrm{T}_{\text {int }}$-tempo de interrupção para efetuar reparos ou manutenção $(h)$.

De acordo com Oliveira et al. (2009), a eficiência operacional é a percentagem do tempo efetivamente trabalhado, em relação ao tempo programado para o trabalho, determinada por meio da Equação 4.

$E O=\frac{T_{p r o}}{T_{p r o}+T_{i m p}} 100$

em que:

Eo- eficiência operacional (\%);

$\mathrm{T}_{\text {pro }}$ - tempo produtivo (h);

$\mathrm{T}_{\text {imp }}$-tempo improdutivo (h).

A velocidade média de deslocamento foi determinada, cronometrando-se o tempo necessário para percorrer cada plano horizontal de trabalho. A estimativa da capacidade de campo foi fundamentada no método proposto por Mialhe (1974). Para determinar a capacidade de campo efetiva, ponderou-se a largura e a velocidade efetiva de trabalho (Equação 5). A capacidade de campo operacional foi obtida por meio dos tempos despendidos com as manobras e as atividades auxiliares à operação (Equação 6).

$C_{c} E=\frac{A}{T_{p r o}}$

em que:

$\mathrm{C}_{\mathrm{c}} \mathrm{E}$ - capacidade de campo efetiva $\left(\mathrm{ha}^{-1}\right)$;

A - área irrigada (ha);

$\mathrm{T}_{\text {pro }}$ - tempo produtivo (h);

$C_{c} O=\frac{A}{T_{p r o}+T_{m}+T_{a u x}}$

em que:

$\mathrm{C}_{\mathrm{c}} \mathrm{O}$ - capacidade de campo operacional $\left(\mathrm{ha}^{-1}\right)$;

A - área irrigada (ha);

$\mathrm{T}_{\text {pro }}$ - tempo produtivo (h);

$\mathrm{T}_{\mathrm{m}}$ - tempo despendido com as manobras (h);

$\mathrm{T}_{\text {aux }}$ - tempo auxiliar despendido com o abastecimento do tanque de fertilizante, interrupções operacionais, manutenção e necessidades fisiológicas (h).

Para estimar a perda de área trabalhada, em decorrência dos tempos despendidos com interrupções operacionais e não operacionais, foi obtido o percentual de rendimento de campo efetivo por meio da Equação 7. 
$R_{c} E=\frac{C_{c} O}{C_{c} E} 100$

em que:

$\mathrm{R}_{\mathrm{c}} \mathrm{E}$ - rendimento de campo efetivo (\%);

$\mathrm{C}_{\mathrm{c}} \mathrm{O}$ - capacidade de campo operacional $\left(\mathrm{ha}^{-1}\right)$;

$\mathrm{C}_{\mathrm{c}} \mathrm{E}$ - capacidade de campo efetiva $\left(\mathrm{ha}^{-1}\right)$.

O delineamento experimental empregado foi o inteiramente casualizado (DIC), constituído por 2 tratamentos, com 6 repetições cada um. Os resultados obtidos foram submetidos à análise de variância e comparados por meio do teste F, admitindo-se 5\% de probabilidade de erro, por meio do software estatístico R ( $R$ Development Core Team, 2011).

Os custos operacionais foram estimados por meio do método proposto pela American Society of Agricultural Engineers (ASAE, 2001) e foram expressos em dólar comercial americano, oficial do Banco Central do Brasil (PTAX 800), a preço de venda (Banco Central do Brasil, 2010), por hora de trabalho $\left(\mathrm{US} \$ \mathrm{~h}^{-1}\right.$ ). Foi considerado como taxa de câmbio o preço da moeda estrangeira, medido em unidades e frações da moeda nacional, que era de $\mathrm{R} \$ 1,6554$ (13/10/2010). Os custos operacionais englobaram os custos fixos de depreciação (linear), juros simples, abrigo, taxas e seguros, sendo considerado o custo do conjunto (trator+implemento). Os custos variáveis foram de combustíveis (volumétrico), lubrificação, mão de obra e manutenção. Foi considerada uma taxa de juros de $10 \%$ a.a. e vida útil de 5 anos, com um valor residual de $49 \%$. O custo de produção (US\$ ha $\mathrm{h}^{-1}$ ) foi obtido por meio da divisão do custo operacional do conjunto, pela capacidade de campo operacional.

O rendimento energético foi obtido pela razão entre o consumo específico efetivo de combustível e a capacidade de campo efetiva. O consumo horário de combustível volumétrico (Chv) foi determinado, utilizando-se um medidor de fluxo instalado no sistema de alimentação do trator agrícola, de acordo com o descrito por Cordeiro (2000), conforme a Equação 8. Os pulsos gerados pelo medidor foram convertidos em volume, considerando-se a frequência de $1 \mathrm{~mL}$ por pulso.

$C_{h v}=\frac{N p 3,6}{t}$

em que:

$\mathrm{C}_{\mathrm{hv}}$ - consumo horário de combustível volumétrico $\left(\mathrm{L} \mathrm{h}^{-1}\right)$;

$\mathrm{Np}$ - número de pulso do medidor de combustível;

$\mathrm{t}$ - tempo de percurso na parcela.

Para o cálculo do consumo específico efetivo (CSE), foi utilizado o método proposto pela United Nations
Economic Commission for Europe (UNECE, 2007), descrita na Equação 9.

$C S E=\frac{G_{f}}{P_{e}}$

em que:

CSE - consumo específico efetivo $\left(\mathrm{g} \mathrm{kW}^{-1} \mathrm{~h}^{-1}\right)$;

$\mathrm{G}_{\mathrm{f}}$-consumo horário em carburante $\left(\mathrm{g} \mathrm{h}^{-1}\right)$;

$\mathrm{P}_{\mathrm{e}}$ - potência efetiva $(\mathrm{kW})$.

O rendimento energético foi obtido de acordo com Lopes (2007) conforme a Equação 10.

$R E=\frac{C S E}{C_{c} O}$

em que:

$\mathrm{RE}$ - rendimento energético $\left(\mathrm{g} \mathrm{kW} \mathrm{ha}^{-1}\right)$;

CSE - consumo específico efetivo $\left(\mathrm{g} \mathrm{kW}^{-1} \mathrm{~h}^{-1}\right)$;

$\mathrm{C}_{\mathrm{c}} \mathrm{O}$ - capacidade de campo operacional (ha $\left.\mathrm{h}^{-1}\right)$.

\section{RESULTADOS E DISCUSSÃO}

Foi estabelecido, para um erro de amostragem admissível em 5\%, o número mínimo de 669 ciclos operacionais. Durante o estudo, foram observados 972 ciclos, número superior ao fixado como necessário, em uma área total de 31,87 hectares.

Seixas et al. (2004) consideram o estudo de tempos e movimentos uma técnica muito importante no desenvolvimento de operações florestais, pois o tempo consumido para cada um dos elementos do ciclo operacional permite a organização do trabalho, deduzir a produtividade e o custo por unidade produzida. $O$ problema de avaliação do tempo foi discorrido por Steiner (1959), Becker (1965) e Harrison \& Quarmby (1972), sem, contudo, chegarem a um consenso sobre qual é o melhor método para avaliálo. O método adotado para o estudo de tempos e movimentos foi o mesmo adotado por Valverde (1995) e Moreira (2000), o qual possibilita constatar os percentuais dos tempos despendidos nas etapas dos ciclos operacionais e a constituição média das atividades parciais, conforme apresentada na Figura 1.

Dentre as atividades parciais efetivas que compuseram o ciclo operacional, é possível observar que o tempo despendido para a atividade parcial "irrigar", em ambos os tratamentos, foi superior a $40 \%$ do ciclo operacional da irrigação pós-plantio de eucalipto no campo, seguido do tempo auxiliar para realização de manobras, que consumiu 20,83 e 27,51\% do tempo total para os tratamentos 1 e 2 , respectivamente. A atividade parcial "abastecer tanque pipa" representou, em média, 9\% do tempo total do ciclo operacional. Dentre as atividades parciais que integram o 
tempo improdutivo, estão as interrupções operacionais necessárias para o reabastecimento do tanque pipa, a qual consumiu em torno de $15 \%$, as atividades para necessidades fisiológicas, que representaram 3,5\% do tempo total do ciclo operacional, e a manutenção com $5 \%$ sendo a única que diferiu estatisticamente entre os tratamentos (valor de F = 24,135; -valor $<0,01$ ).

Na Tabela 1, pode ser observado que houve uma diferença estatística para a disponibilidade mecânica, fato esse explicado pelo maior tempo despendido para efetuar a manutenção corretiva do trator agrícola, durante a operação no tratamento 1 , originando uma diminuição da eficiência de utilização e operacional, as quais também diferiram estatisticamente. Os percentuais de eficiência operacional ficaram abaixo dos encontrados por outros autores, ASAE (1999), Molin \& Milan (2002) e Silveira (2001), que obtiveram em seus estudos percentuais entre 70 e $90 \%$ de eficiência operacional, corroborando Machado et al. (1996) os quais afirmam que a manutenção adequada das máquinas aumenta a eficiência do trabalho, minimizando perdas de tempo com paradas para correção de eventuais problemas.

Na Tabela 2, são apresentados os resultados das determinações de capacidade de trabalho e de eficiência de cam- po. A velocidade média de deslocamento, o consumo horário de combustível e a capacidade de campo efetiva foram estatisticamente semelhantes entre os tratamentos. A menor $\mathrm{CcO}$ foi obtida no tratamento $2(36,55 \%)$ diferindo estatisticamente pelo teste $\mathrm{F}$. O custo de produção comportouse inversamente proporcional à produtividade da máquina agrícola, ou seja, houve uma relação contrária de acordo com a capacidade de campo operacional, os quais diferiram estaticamente entre si. O conhecimento e aplicação de critérios técnicos e econômicos para obtenção dos custos de produção são de suma importância para o setor agroflorestal, pois permitem que os custos estimados apresentem solidez e sejam confiáveis, proporcionando, dessa forma, uma melhor competitividade de mercado. Peloia \& Milan (2010) complementam que as organizações líderes, dos mais diferentes setores, têm como característica medir o próprio desempenho de modo sistêmico, porém, nas empresas agrícolas ainda não é comum o emprego desse conceito, incluindo o setor de mecanização. Em relação ao rendimento energético, decorrente de uma função direta da $\mathrm{CcE}$, esse comportou-se de forma análoga, não diferindo estatisticamente pelo teste $\mathrm{F}$.

O somatório dos custos fixos e variáveis resultou em custo operacional de US\$31.95 por hora de trabalho. Na

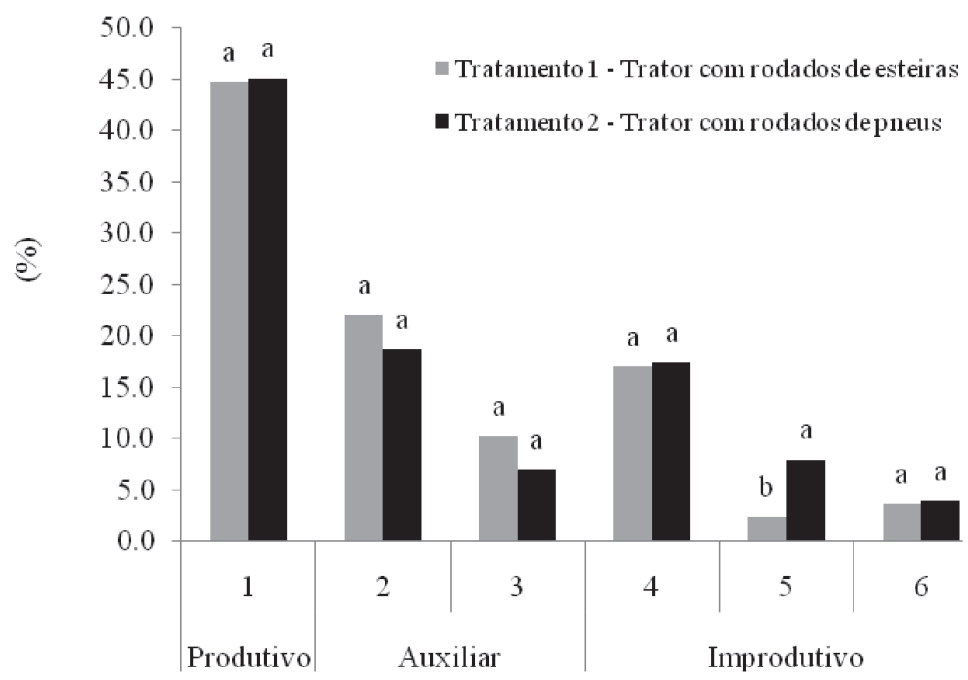

(1)- Irrigar; (2) - Manobras; (3) - Abastecer tanque pipa; (4) - Interrupções operacionais; (5) - Manutenção; (6) - Tempo pessoal.

Figura 1. Composição percentual do tempo total do ciclo operacional da irrigação pós-plantio de eucalipto a campo.

Tabela 1. Estimativa da disponibilidade mecânica, eficiência de utilização e eficiência operacional

\begin{tabular}{lccc}
\hline Tratamentos & $\begin{array}{c}\text { Disponibilidade mecânica } \\
(\boldsymbol{\%})\end{array}$ & $\begin{array}{c}\text { Eficiência de utilização } \\
(\boldsymbol{\%})\end{array}$ & $\begin{array}{c}\text { Eficiência operacional } \\
(\boldsymbol{\%})\end{array}$ \\
\hline 1 & 98,04 & 80,27 & 61,99 \\
2 & 92,08 & 72,48 & 50,49 \\
\hline C.V. (\%) & 3,66 & 8,14 & 16,46 \\
Valor de F & 8,789 & 6,261 & 4,631 \\
p-valor & 0,000 & 0,000 & 0,005 \\
\hline
\end{tabular}

C.V.(\%): coeficiente de variação.

Rev. Ceres, Viçosa, v. 59, n.2, p. 164-170, mar/abr, 2012 
Figura 2, é apresentado o gráfico em percentuais dos custos operacionais obtidos. Os custos fixos (depreciação, juros simples, abrigo, taxas e seguros) corresponderam a $14,95 \%$ e os custos variáveis (combustível, reparos e manutenção, lubrificação e mão de obra) representaram $85,05 \%$. O custo de combustível foi o que mais pesou nos custos operacionais $(39,56 \%)$, o qual, fundamentalmente, representa entre 35 e $45 \%$ do custo total de produção
(Ibañes \& Rojas, 1994), seguido do custo de mão de obra que representou aproximadamente $35 \%$.

$\mathrm{Na}$ Figura 3, pode ser observada uma curva do tipo exponencial, relacionando o custo de produção (US\$ ha $\mathrm{h}^{-1}$ ) com a CcO. O custo de produção variou de US\$131.63, para uma $\mathrm{CcO}$ de $0,20 \mathrm{ha} \mathrm{h}^{-1}$, a uma faixa de melhor $\mathrm{CcO}$, de 1,10 ha h$^{-1}$, a US\$23.93, na faixa de melhores condições da operação de irrigação pós-plantio de eucalipto a campo.

Tabela 2. Valores médios de velocidade (VM), consumo horário de combustível volumétrico (Chv), capacidade de campo efetiva (CcE), capacidade de campo operacional $(\mathrm{CcO})$, rendimento de campo efetivo $(\mathrm{RcE})$, custo de produção $(\mathrm{CP})$ e rendimento energético $(\mathrm{RE})$

\begin{tabular}{|c|c|c|c|c|c|c|c|}
\hline Tratamentos & $\begin{array}{c}\text { VM } \\
\left(\mathrm{km} \mathrm{h}^{-1}\right)\end{array}$ & $\begin{array}{c}\text { Chv } \\
\left(\mathbf{L} \mathbf{h}^{-1}\right)\end{array}$ & $\begin{array}{c}\text { CcE } \\
\left(h^{-1} \mathbf{h}^{-1}\right)\end{array}$ & $\begin{array}{c}\mathrm{CcO} \\
\left(\mathbf{h a ~ h}^{-1}\right)\end{array}$ & $\begin{array}{r}\text { RcE } \\
(\%)\end{array}$ & $\begin{array}{c}\text { CP } \\
\left(\mathrm{US} \$ \mathrm{ha} \mathrm{h}^{-1}\right)\end{array}$ & $\begin{array}{c}\mathbf{R E} \\
\left(\mathrm{g} \mathrm{kW} \mathbf{h a}^{-1}\right)\end{array}$ \\
\hline 1 & 3,20 & 9,3 & 0,95 & 0,46 & 50,22 & 58,63 & 151,23 \\
\hline 2 & 3,24 & 9,5 & 0,97 & 0,36 & 36,55 & 81,56 & 158,41 \\
\hline C.V. $(\%)$ & 28,07 & 11,47 & 28,07 & 24,33 & 21,44 & 23,15 & 22,78 \\
\hline Valor de F & 0,006 & 0,214 & 0,016 & 3,322 & 6,484 & 10,914 & 0,095 \\
\hline p-valor & 0,940 & 0,654 & 0,263 & 0,009 & 0,000 & 0,002 & 0,764 \\
\hline
\end{tabular}

C.V.(\%): coeficiente de variação.

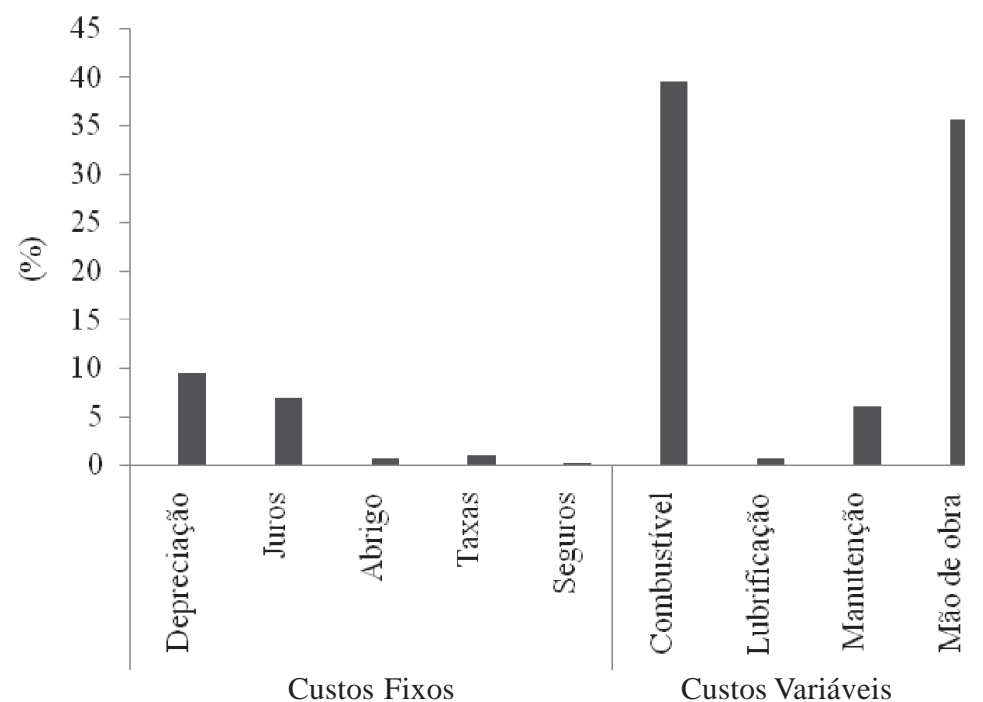

Figura 2. Percentuais dos custos fixos e variáveis do trator agrícola.

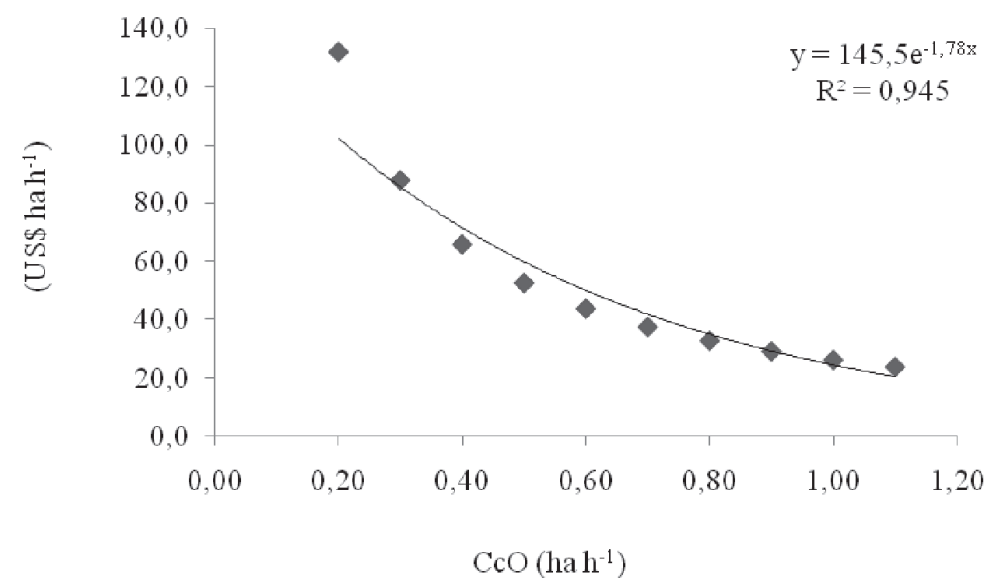

Figura 3. Comportamento do custo de produção em função da $\mathrm{CcO}$. 


\section{CONCLUSÕES}

Em áreas ocupadas anteriormente por reflorestamentos de eucalipto com distintos números de reforma, a capacidade de campo efetiva da irrigação pós-plantio de eucalipto a campo não diferiu estatisticamente.

O tempo improdutivo exerceu impacto sobre a capacidade de campo operacional e, consequentemente, aumentou o custo de produção em aproximadamente $30 \%$.

\section{REFERÊNCIAS}

American Society of Agricultural Engineers (2001) ASAE standards 2001: machinery, equipment, and buildings: operating costs. Ames, Iowa, USA, 164:226. ASAE D472-3.

American Society of Agricultural Engineers (1999) ASAE Standards 1999.Agriculturalmachinery management data. St. Joseph, USA, 332:339. ASAE D497-2.

Banco Central do Brasil. Conversão de moedas. Disponível em: <http://www4.bcb.gov.br/pec/conversao/conversao.asp>. Acessado em: 13 janeiro de 2011.

Becker GS (1965) A theory of allocation of time. Economic Journal, 75: 493-517.

Barnes RM (1968) Motion and time study: design and measurement of work. 6 ed. New York: John Willey \& Sons, 799p.Becker GS (1965) A theory of allocation of time.Economic Journal, 75: 493-517.

Buzetto FA, Bizon JMC\& Seixas F(2002) Avaliação de polímero absorvente à base de acrimalida no fornecimento de água para mudas de Eucalyptus urophylla em pós-plantio. Piracicaba, IPEF. 8p. (Circular técnica, 192).

Cordeiro MAL (2000) Desempenho de um trator agrícola em função do pneu, da lastragem e da velocidade de deslocamento. Tese de Doutorado. Universidade Estadual Paulista, Botucatu 153p.

Empresa Brasileira de Pesquisa Agropecuária- EMBRAPA (1999) Sistema Brasileiro de Classificação de Solos. Rio de Janeiro, Centro Nacional de Pesquisa de Solos. 412p.

Gatto A, Barros NF, Novais R F, Costa LM\& Neves JCL (2003)Efeito do método de preparo do solo, em área de reforma, nas suas características, na composição mineral e na produtividade de plantações de Eucalyptus grandis. Revista Árvore, 27:635-646.

Gruber YBG (2006) Otimização da lâmina de irrigação na produção de mudas clonais de eucalipto (Eucalyptusurophylla $\mathrm{x}$ Eucalyptus grandis e Eucalyptusurophyllavar.plathyphylla). Dissertação de Mestrado. Escola Superior de Agricultura "Luiz de Queiroz", 144p.

Harrison AJ, Quarmby da (1972) The value of time. In: Layard R (Ed.) Cost-Benefit Analysis. London, Penguin, p.173-208.

Ibañez M \& Rojas E (1994) Costos de operación y producción por concepto de maquinaria agrícola. Concepción, Universidad Concepción. 58 p.

Lima JEFW, Ferreira RSA\&Christofidis D (2004) O uso da irrigação no Brasil. Disponível em: <http://www.cf.org.br/cf2004/ concurso.php>. Acessado em: 02 jul. 2009.

Lopes SE (2007) Análise técnica e econômica de um sistema de colheita florestal. Tese de Doutorado. Universidade Federal de Viçosa, Viços,. 124p.

Machado ALT, Reis AV, Moraes MLB, Alonço AS (1996) Máquinas para preparo do solo, semeadura, adubação e tratamentos culturais. Pelotas, UFPel. 229p.
Mialhe LG (1974) Manual de mecanização agrícola. São Paulo, Agronômica Ceres. 301p.

Molin JP \& Milan M (2002) Trator-implemento: dimensionamento.Capacidade operacional e custo.In: Gonçalves, J. L. M.; Stape, J. L. (Org.) Conservação e cultivo de solos para plantações florestais. Piracicaba, Instituto de Pesquisas Florestais, p.409-436.

Molin JP, Milan M,Nesrallah MGT, Castro CN \& Gimenez LM (2006) Utilização de dados georreferenciados na determinação de parâmetros de desempenho em colheita mecanizada. Revista Engenharia Agrícola. 26:759-767.

Moreira FMT (2000) Análise técnica e econômica de subsistemas de colheita de madeira de eucalipto em terceira rotação. Dissertação de Mestrado Universidade Federal de Viçosa, Viçosa, 148p.

Oliveira D, Lopes ES, Fiedler NC (2009) Avaliação técnica e econômica do forwarder em extração de toras de pinus. Scientia Forestalis, 37:525-533.

Peloia PR \&Milan M (2010) Proposta de um sistema de medição de desempenho aplicado à mecanização agrícola. Revista Engenharia Agrícola, 30:681-69.

R Development Core Team (2011) A language and environment for statistical computing. $\mathrm{R}$ Foundation for StatisticalComputing, Vienna.

Seixas F, Barbosa RF, Rummer R (2004) Tecnologia protege saúde do operador. Revista da Madeira, 14:68-73.

Silva KR, Minetti LJ, Fiedler NC, Venturoli F, Machado EGB \& Souza AP (2004) Custos e rendimentos operacionais de um plantio de eucalipto em região de cerrado. Revista Árvore, 28:361-366.

Silveira GM (2001) Os cuidados com o trator. $1^{\mathrm{a}}$.ed. Viçosa, Aprenda Fácil Editora, 309p.

Simões D, Fenner PT (2010) Avaliação técnica e econômica do forwarder na extração de madeira em povoamento de eucalipto de primeiro corte. Revista Floresta, 40:711-720.

Simões, D Silva MR (2010) Análise técnica e econômica das etapas de produção de mudas de eucalipto. Revista Cerne, 16:359366.

Simões, JW (1989) Reflorestamento e manejo de florestas implantas. Piracicaba, Escola Superior de Agricultura "Luiz de Queiroz". 29p.

Steiner P (1959) Choosingamongalternativeinvestment in thewater-resoSurcefield. American Economic Review, 49:893919.

Superintendência de Estudos Econômicos e Sociais da Bahia BAHIA (1998) Atributos climáticos do Estado da Bahia. Salvador. $85 \mathrm{p}$.

United Nations Economic Commission for Europe - UNECE (2007) $63^{\text {rd }}$ session: Fuel consumption for vehicle powered refrigeration units based on a conception of standard vehicle engine. INF.3, Geneva. 160p.

Valverde SR (1995) Análise técnica e econômica do subsistema de colheita de árvores inteiras em povoamentos de eucalipto. Dissertação de Mestrado Universidade Federal de Viçosa, Viçosa $123 \mathrm{p}$. 